\title{
Review: complex organisational and educational interventions appear to be effective for managing depression in primary care
}

\author{
Gilbody S, Whitty P, Grimshaw J, et al. Educational and organizational interventions to improve the management of \\ depression in primary care: a systematic review. JAMA 2003;289:3145-51.

\section{QUESTION: What is the effectiveness of organisational and educational interventions for the management of depression in primary care settings?}

\section{Data sources}

Studies were identified by searching Medline, PsycLIT, EMBASE/Excerpta Medica, CINAHL, the Cochrane Controlled Trials Register, the UK National Health Service Economic Evaluations Database, the Cochrane Depression Anxiety and Neurosis Group register, and the Cochrane Effective Professional and Organisational Change (EPOC) Group specialist register (all from inception to March 2003); contacting experts; and scanning bibliographies of included studies.

\section{Study selection}

Studies were selected if they were randomised or non-randomised controlled trials, controlled before and after studies, or interrupted time series studies; examined organisational or educational interventions targeted at primary healthcare professionals and patients for the management of depression; and assessed depression, health related quality of life, and cost outcomes. Studies were excluded if they examined the efficacy of patient level interventions only or screening strategies for depression only.

\section{Data extraction}

Data were extracted on setting, study design, methodological quality, interventions, outcomes, follow up period, and results.

\section{Main results}

36 studies were selected; 29 were randomised or non-randomised controlled trials, 5 were controlled before and after studies, and 2 were interrupted time series studies. Most studies were done in US primary care practices. Studies were heterogeneous, with many using multifaceted interventions including guidelines (22 studies), changes in delivery system design (19 studies), attention to the information needs of patients (11 studies), ready access to necessary expertise (21 studies), and information support systems (10 studies). 19 studies were randomised by clinician or clinical practice. 21 studies had positive results for the primary outcomes.

2 randomised studies examined complex organisational and educational interventions of patient screening, clinician education, patient specific reminders, nurse case management, and integration with specialist care. They reported improved medication adherence and depression outcomes at 6 and 12 months; at 24 months, improved medication adherence and global outcome persisted but benefits on depression outcomes did not.

Studies showed that depression outcomes improved with case management by primary care nurses, practice counsellors, or graduate psychologists. In these studies, the nurse or trained counsellor were involved in either brief patient education and medication counselling or telephone support, or nurse case management was a core component of a complex strategy (eg, patient education, and ongoing support and monitoring).

Guidelines and strategies to implement them were generally ineffective unless educational interventions were accompanied by complex organisational interventions, such as nurse case management or collaborative care.

\section{Conclusions}

Most studies examining the effectiveness of organisational and educational interventions for the management of depression in primary care settings use multifaceted interventions. Complex organisational and educational interventions, or the enhanced involvement of nurses or trained counsellors in case management, appear to improve depression outcomes. The use of simple guidelines is generally ineffective.
Sources of funding:

United Kingdom

Medical Research

Council; Wellcome

Health Services

Research Fellowship

Programs; United

Kingdom National

Health Service Centre

for Reviews and

Dissemination; United

Kingdom Health

Technology Assessment

Programme.

For correspondence:

Dr S Gilbody,

University of Leeds,

Leeds, UK.

s.m.gilbody@leeds.ac.uk

A modified version of the abstract appears in

Evidence-Based

Nursing.

\section{COMMENTARY}

The US Preventive Services Task Force currently recommends screening adults for depression in primary care settings with the following caveat: such screening is effective only in clinical practices that have systems in place to assure accurate diagnosis, effective treatment, and follow up. ${ }^{1}$ Gilbody et al underscore the importance of this caveat with regard to diagnosis and management of depression in the primary care arena.

In this review, Gilbody et al note that educational programmes by themselves are often ineffective in helping providers appropriately identify and treat depression. Thus, although provider training and background are necessary for optimal care, ${ }^{2}$ they are not sufficient for it. Still, all primary care providers should screen with an appropriate test ${ }^{3}$ and manage depression according to an accepted guideline. ${ }^{4}$

Consistent with previous research, ${ }^{5}$ the authors find that complex, systems based approaches such as collaborative care, nurse case management, and intensive quality improvement are effective. The Robert Wood Johnson programme Depression in primary care: linking clinical and systems strategies is a current national effort studying the feasibility of integrating such organisational depression management tools into primary care settings. ${ }^{5}$

Unless managed care organisations provide reasonable financial and non-financial incentives, however, medical practices are unlikely to adopt complex interventions. ${ }^{5}$ Such incentives may include either direct reimbursement for appropriate screening procedures or payment for the improved clinical outcomes that should accompany rigorous, systems based depression screening and management.

Brian A Primack, MD, EdM

Harold Alan Pincus, MD University of Pittsburgh Medical Center Pittsburgh, Pensylvania, USA

1 US Preventive Services Task Force. Screening for depression recommendations and rationale. Ann Intern Med 2002;136:760-4.

2 Williams JW Jr, Rost K, Dietrich AJ, et al. Primary care physicians' approach to depressive disorders. Effects of physician specialty and practices phy this patient clinically depressed? JAMA 2002;287:1160-70.

4 Schulberg HC, Katon W, Simon GE, et al. Treating major depression in primary care practice: an update of the Agency for Health Care Policy and Research Practice Guidelines. ;5:1121-7.

5 Pincus HA, Pechura CM, Elinson L, et al. Depression in primary care: linking clinical and systems strategies. Gen Hosp Psychiatry 2001;23:311-8. 